\title{
Correlation between Calorie Intake and Nutritional Status of Autism Spectrum Disorder in Children
}

\author{
Aryo Windaru, ${ }^{1}$ Fifi Veronica, ${ }^{2}$ Dian Marta Sari ${ }^{3}$ \\ ${ }^{1}$ Faculty of Medicine Universitas Padjadjaran, ${ }^{2}$ Department of Anatomy and Cell Biology Faculty \\ of Medicine, Universitas Padjadjaran, ${ }^{3}$ Department of Physical Medicine and Rehabilitation \\ Faculty of Medicine Universitas Padjadjaran/Dr. Hasan Sadikin General Hospital Bandung
}

\section{Abstract}

Background: Autism Spectrum Disorder (ASD) is a severe pervasive developmental disorder with prevalence as high as one in sixty-eight children. Children diagnosed with ASD may have food intake problem and might affect their nutritional status in the future. The objective of this study was to analyze the correlation between total calorie intake and nutritional status of ASD children.

Methods: This cross-sectional study was conducted in Indigrow Child Development and Autism Center involving 16 patients from October to November 2015. Total calorie intake was assessed by 24-hour food recall and nutritional status was measured by Z-score. Correlation was analyzed using Spearman's Rho.

Results: Overweight and obesity were found in 10 out of 16 ASD children assessed. Total calorie intake was not significantly correlated with nutritional status of ASD children $(r=0.021, p=0.940)$.

Conclusions: There is no significant relevance between total calorie intake and nutritional status in ASD children at Indigrow Child Development and Autism Center. [AMJ.2016;3(2):319-22]

Keywords: Autism, calorie intake, nutritional status, Z-score

\section{Introduction}

Autism Spectrum Disorder (ASD) is a neurodevelopmental disability that is usually diagnosed before 3 years of age and is characterized by deficits in social reciprocity and in language skills that are associated with repetitive behaviours and restricted interests. ${ }^{1}$ According to Centers for Disease Control and Prevention (CDC), about 1 in 68 children identified with ASD. ${ }^{2}$

Rogers et al. ${ }^{2}$ reported that $46-75 \%$ children specifically diagnosed with ASD have feeding, eating, and mealtime challenges. Some study discovered that children with autism have anomalous habits relating to food and eating. These children are restricted by food category and texture, and also refused food more. Weighing from the risk, feeding problems might affect the nutritional status of ASD children themselves. ${ }^{3}$

One study conducted in Japan ${ }^{4}$ with 140 samples of Japanese children aged 7-18 years with autism discovers $25 \%$ obesity among those subjects. Another study supports this evidence, Curtin et al. ${ }^{5}$ concluded children with autism have $40 \%$ higher risk in suffering from obesity. Overweight and obesity are not trivial issues considering the effect in increasing mortality of humankind. Various complication may happen in consequence of obesity which are type 2 diabetes mellitus, hypertension, dyslipidaemia, and nonalcoholic steatohepatitis. ${ }^{6}$ Despite of these concerns, scarcity in research related to nutritional status of ASD children is still a problem. ${ }^{1}$ This study proposed hypothesis of correlation between nutritional intake and nutritional status on ASD children in Indigrow Children Development \& Autism Center, Bandung.

\section{Methods}

Research design used in this study was crosssectional. Nutritional intake as independent variable was obtained by conducting interview to the parents of ASD patients who came to the clinic. Subjects included in this study were children with ASD from 2 to 17 years old at

Correspondence: Aryo Windaru, Faculty of Medicine, Universitas Padjadjaran, Jalan Raya Bandung-Sumedang Km.21, Jatinangor, Sumedang, Indonesia, Phone: +62 85693260065 Email: aryowindaru@gmail.com 
Table 1 Nutritional Status based on Z-score

\begin{tabular}{lc}
\hline \multicolumn{1}{c}{ Category } & Z-score \\
\hline Obese & $>2$ SD \\
Overweight & $>1$ to 2 SD \\
Normal & $\geq-2$ SD to $\leq 1$ SD \\
Underweight & $\geq-3$ SD to $<-2$ SD \\
Severe Underweight & $<-3$ SD \\
\hline Note: Based on Keputusan Menteri Kesehatan (Kepmenkes) Indonesia No: $1995 /$ Menkes/SK/XXI/2010
\end{tabular}

Indigrow Child Development and Autism Center from October to November 2015. There were 16 children who met the inclusion criteria. Every parent or guardian was given an informed consent about the procedures of this research and asked about the willingness to participate in this study. Study was conducted after obtaining Ethical Clearance No: 485/UN.C1.3.2/KEPK/PN/2015 from Health Research Ethics Committee, Faculty of Medicine, Universitas Padjadjaran.

Calorie intake was acquired from 24hour food recall by interviewing the parents of ASD children regarding foods and drinks consumed a day before. Two separate interviews were necessary to acquire calorie intake in weekdays and weekends to minimize bias. Calorie conversion guideline used in this study was Indonesian Food Composition Table (Indonesian FCT) which acquired from Department of Medical Nutrition, Faculty of Medicine, Universitas Padjadjaran.

Nutritional status was measured in Z-score, a scoring system to assess differences of child growth compared to references in the same age and gender. Reference values of
Z-score and body mass index (BMI) in this study were taken from Keputusan Menteri Kesehatan (Kepmenkes) Indonesia No: 1995/ Menkes/SK/XXI/2010 tentang Standar Antropometri Penilaian Status Gizi Anak. Z-score was calculated after taking height and weight measurement at the first visit which transformed into BMI. Z-score was acquired by calculating BMI together with age and gender. Date of birth was taken in order to determine the age of ASD patients at the first visit. Correlation between calorie intake and Z-score was analysed using Spearman's Rho regarding the calorie intake was not normally distributed in Shapiro-Wilk test ( $p=0.003)$.

\section{Results}

Male participants were higher than female participants (14 out of 2 children). Frequency of overweight and obesity among ASD children in Indigrow Child Development and Autism Center was 10 out of 16 children.

Spearman's Rho correlation test shows no significance of correlation between calorie

Table 2 Characteristics of ASD Children Aged 2-17 years at Indigrow Child Development and Autism Center

\begin{tabular}{lcc}
\hline \multicolumn{1}{c}{ Characteristics } & Mean (SD) or Median (Min-Max) & Frequency (n=16) \\
\hline Gender & & 14 \\
Males (n) & $1811.94(1174.24-5073.95)$ & 2 \\
Female (n) & $2.76(0.74)$ & 16 \\
Total calorie per day (kcal) & 16 \\
Z-score & 0 \\
Underweight (n) & 6 \\
Normal (n) & 3 \\
Overweight (n) & 7 \\
Obese (n) & \\
\hline
\end{tabular}


intake and Z-score $(\mathrm{r}=0.021, \mathrm{p}=0.940)$.

\section{Discussion}

This study showed that 14 of 16 participants were male, this results congruent with a previous study showing that autism occurs 4 to 5 times more in males compared to females. ${ }^{7}$

Seven out of 16 participants were obese, but this study found that calorie intake was inconsistent with nutritional status. This phenomena can be explained in the way of obesity caused by multifactorial factors not just by a single etiology., ${ }^{8,9}$ Other factors like physical activities, genetics, psychotropic drugs, and sleep hygiene may also contribute in manufacturing obesity. The most common genetic alteration associated with ASD is genomic duplications and deletions at 16 11.2 gene. Deletion in this region has recently been shown to play role in earlyonset childhood obesity. The pediatric carriers of $16 \mathrm{p} 11.2$ are $50 \%$ obese. Psychotropic drugs are very commonly prescribed in children with ASD. Studies about children with ASD treated by risperidone discovered significant weight gain versus placebo groups. Yet the mechanisms by which antipsychotics cause weight gain are not fully understood. Children with ASD are often faced by sleep problems. Several studies documented that overweight in children is inversely correlated with sleep duration. Physical activity and sedentary behaviour are factors which contributed in obesity development of children and adolescent. Nonetheless, children with ASD may be particularly challenged to engage in physical activity in consideration of motor skill difficulties. ${ }^{8}$

Evans et al. ${ }^{10}$ stated that ASD children have higher levels consumption of energy dense foods than typically developing children. Most calorie intake of ASD children in this study consists of snacks or instant noodles (14 out of 16) according to the 24-hour food recall and 6 out of 16 patients have higher calorie diet based on age according to Recommended Dietary Allowances: Harmonization in Southeast Asia.

This study had several limitations; recall bias may have occurred in this study (e.g., mothers' reluctance to report "unhealthy" food intake), which would underestimate the true association between calorie intake and nutritional status.

Methods such as 24-hour recall or food diaries evaluate calorie intakes more accurately, yet estimate short-term not long- term intake. ${ }^{11}$ Some of the ASD children in this study were hardly receive instructions and cooperate in measurement which may affect the measurement accuracy and caused measurement bias.

The largest methodological limitation presented among cross-sectional studies is a confounding factor because measurement of calorie intake only measured in one time only and it is difficult to ascertain whether calorie intake is contributing to nutritional status of ASD children. However, cross-sectional studies are vital in facilitating hypothesis initiation to be further tested in observational or experimental studies. ${ }^{12}$

Prospective cohort studies are better equipped than cross-sectional studies to adjust confounding factors but are still observational in nature and thus vulnerable to confounding factors as well. ${ }^{10}$ Total sampling was used in this study, but still the sample was lacking $(\mathrm{N}=16)$ and may not representative for community.

In conclusion, this study does not show any significant correlation between total calorie intake and nutritional status of ASD in children. This result is consistent with some other studies stating that total calorie intake is statistically insignificant toward nutritional status.

\section{References}

1. Xia W, Zhou Y, Sun C, Wang J, Wu L. A preliminary study on nutritional status and intake in Chinese children with autism. Eur J Pediatr. 2010;169(10):1201-6.

2. Rogers L, Magill-Evans J, Rempel G. Mothers' challenges in feeding their children with autism spectrum disordermanaging more than just picky eating. J Dev Phys Disabil. 2012;24(1):19-33.

3. Nierengarten MB. Managing autism symptoms through nutrition. Contemporary Pediatrics. 2014;31(4):237.

4. Sugiyama T. A research of obesity in autism. Japanese Journal on Developmental Disabilities. 1991;13:53-8

5. Curtin C, Bandini LG, Perrin EC, Tybor DJ, Must A. Prevalence of overweight in children and adolescents with attention deficit hyperactivity disorder and autism spectrum disorders: a chart review. BMC Pediatr. 2005;5:48-53.

6. Batch JA, Baur LA. Management and prevention of obesity and its complications in children and adolescents. Med J Aust. 
2005;182(3):130-5.

7. Lai MC, Lombardo MV, Baron-Cohen S. Autism. Lancet. 2014;383(9920):896910.

8. Hill JO, Wyatt HR, Peters JC. Energy balance and obesity. Circulation. 2012;126(1):12632.

9. Curtin C, Jojic M, Bandini LG. Obesity in children with autism spectrum disorder. Harv Rev Psychiatry. 2014;22(2):93-103.

10. Evans EW, Must A, Anderson SE, Curtin C, Scampini R, Maslin M, et al. Dietary patterns and body mass index in children with autism and typically developing children. Res Autism Spectr Disord. 2012;6(1):399-405.

11. Dehghan M, Akhtar-Danesh N, Merchant AT. Childhood obesity, prevalence and prevention. Nutr J. 2005;4:24.

12. Rosenheck R. Fast food consumption and increased calorie intake: a systematic review of a trajectory towards weight gain and obesity risk. Obes Rev. 2008;9(6):53547. 